\title{
Lumbar Meningocele
}

National Cancer Institute

\section{Source}

National Cancer Institute. Lumbar Meningocele. NCI Thesaurus. Code C101211.

A congenital abnormality in the lumbar region of the spine in which the meninges

protrude through a defect in the spinal column. 